\title{
Dynamic MAC Parameters Configuration for Performance Optimization in 802.11e Networks
}

\author{
Luca Scalia, Ilenia Tinnirello \\ Universita' di Palermo, Dipartimento di Ingegneria Elettrica \\ Viale delle Scienze, 90128, Palermo, Italy \\ luca.scalia@tti.unipa.it,ilenia.tinnirello@tti.unipa.it
}

\author{
Juki Wirawan Tantra, Chuan Heng Foh \\ School of Computer Engineering \\ Nanyang Technological University, Singapore \\ jw.tantra@ieee.org, aschfoh@ntu.edu.sg
}

\begin{abstract}
Quality of service support in wireless LAN is a theme of current interest. Several solutions have been proposed in literature in order to protect time-sensitive traffic from best-effort traffic. According to the EDCA proposal, which is a completely distributed solution, the service differentiation is provided by giving probabilistically higher number of channel accesses to stations involved in real-time applications. To this purpose, the MAC parameter settings of each contending stations can be tuned dynamically. In this paper, we face the problem of tuning the EDCA MAC parameters in common scenarios in which a given number of low-rate delay-sensitive traffic flows share the channel with some stations involved in data transfer.

Our contribution is threefold. First, we show that, whenever possible, the delay constraints of the high priority class can be satisfied in both the cases of contention windows differentiation and inter-frame space differentiation. However, these mechanisms have different side effects in terms of bandwidth availability for the best effort stations. Second, we propose to exploit the MAC parameter dynamic settings of EDCA in order to probabilistically guarantee the delay requirements and to jointly maximize the aggregated throughput of the network. Finally, we suggest a very simple solution to automate these parameter settings in a real scenario, where traffic flows can be activated/deactivated dynamically, by simply monitoring the channel activity. The proposed solution is very robust, since it does not require any $a$ priori traffic model or any network load estimator.
\end{abstract}

\section{INTRODUCTION}

The Distributed Coordination Function (DCF) of the IEEE 802.11 [1] MAC protocol has received significant research attention due to its robustness and popularity. The protocol is based on the old principle of the Carrier Sense Multiple Access with Collision Avoidance (CSMA/CA) and has been deeply investigated in terms of performance and critical settings [2]. A challenging issue of DCF is the provisioning of service differentiation mechanisms, in order to meet different quality of service requirements for different carried traffic. In fact, the original DCF protocol is long-term fair, and each contenting stations has the same probability to win a transmission grant, without regard to the specific traffic requirements.

Part of the activities carried out in the 802.11e Task Group concerns the proposal of priority mechanisms for the 802.11 DCF aimed at the definition of the Enhanced Distributed Channel Access (EDCA), with mechanisms to support differentiated service [5]. Basically, these mechanisms work on the probabilistic reduction of the backoff counter values (by setting lower contention windows $\mathrm{CWs}$ ), and on the reduction of the inter-frame times AIFSs for the high priority classes.

Great efforts have been done in literature [6], [7], [8], [9] in order to analyze the effects of different prioritization schemes and to identify the most effective settings for a given traffic scenario, with both simulations and analytical models [10], [11]. However, most parts of these studies are limited to some case studies and do not provide general insights to the synthesis of the MAC parameters.

Although the effect of each differentiation parameter, individually taken, can be easily evaluated, evaluation of the combination of various differentiation mechanisms is still lacking. In other words, if we consider a traffic class $c$ with a target desired throughput in a specific network scenario, given that the target QoS can be provided with both the CW and the AIFS differentiations, some aspects need to be clarified:

- what CW settings, for the class $c$ and for the other classes, guarantee probabilistically the target QoS;

- what AIFS settings, for the class $c$ and for the other classes guarantee probabilistically, the target QoS;

- what differences/equivalences exist, for the other network performance figures, between the CW-based and AIFSbased differentiation solutions;

- what optimizations are possible using combinations of the $\mathrm{CW}$ and AIFS parameters, for the same network scenario and QoS target.

Due to the complexity of the medium access modeling, several simplifying assumptions, such as the use of homogeneous and saturated contending stations, do not allow generalization of the results currently provided by the analytical models to answer these questions.

In this work we try to intuitively answer these questions and to provide some guidelines for tuning the per-class MAC parameters in a realistic heterogeneous traffic scenario, in which a given number of low-rate delay-sensitive traffic flows (High Priority HP class) share the channel with some stations involved in data transfer (Low Priority LP class). Because of the complexity of the considered traffic scenario, in order to generalize our conclusions without any simplifying assumption, we base our investigations on simulations. We developed an object-oriented event-driven $\mathrm{C}++$ simulator, where we implemented all the EDCA features. Such simulator has been cross-validated with other 802.11 simulation programs 


\begin{tabular}{|c|c|c|c|c|c|c|}
\hline AC & \multirow{2}{*}{ CWmin } & \multirow{2}{*}{ CWmax } & \multirow{2}{*}{ AIFSN } & \multicolumn{3}{|c|}{ TXOP Limit } \\
\cline { 5 - 7 } & & & DS-CCK & OFDM & Other \\
\hline AC_BK & aCWmin & aCWmax & 7 & 0 & 0 & 0 \\
\hline AC_BE & aCWmin & aCWmax & 3 & 0 & 0 & 0 \\
\hline AC_VI & aCWmin/2 & aCWmin & 2 & $6.016 \mathrm{~ms}$ & $3.008 \mathrm{~ms}$ & 0 \\
\hline AC_VO & aCWmin/4 & aCWmin/2 & 2 & $3.264 \mathrm{~ms}$ & $1.504 \mathrm{~ms}$ & 0 \\
\hline
\end{tabular}

Fig. 1. EDCA default settings

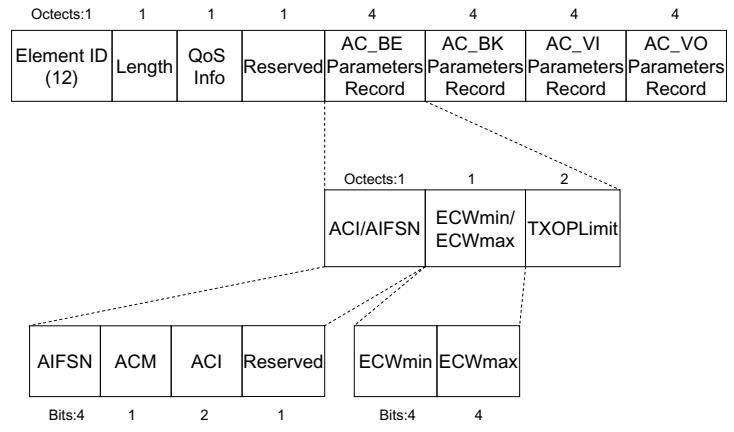

Fig. 2. EDCA Parameter Set Element developed on top of the NS-2 simulator [12] and, when possible, with the analytical models [10] available in the scientific community.

The rest of the paper is organized as follows. In section II, we discuss some significant research works about the 802.11e service differentiation mechanisms. In section III, a brief overview of the EDCA mechanism is provided. In section IV, we discuss the performance results of the coexistence of high priority and low priority stations. In section $\mathrm{V}$, some remarks about the dynamic EDCA MAC parameter tuning are provided. Finally, in section VI some conclusions are drawn.

\section{RELATED WORK}

A large amount of work on service differentiation via distributed mechanisms has been carried out starting from year 2000. The most comprehensive overview of the various complementary as well as mutually exclusive mechanisms for service differentiation in 802.11 is in the technical report [13], which also addresses the performance evaluation of a subset of the considered mechanisms.

Adaptive setting of $\mathrm{CW}$ to achieve better MAC layer performance are considered in [6], [14], which proposes an adaptive algorithm to dynamically re-calculate the $\mathrm{CW}_{\min }$ value according to the specific traffic class and the changes of the network load. [15] also discusses the problem of supporting a distributed admission control rule on top of an enhanced version of DCF, via the definition of a Virtual MAC algorithm, that passively monitors the radio channel and estimates the service levels available, plus a Virtual Source algorithm that used the above mentioned algorithm to adapt application parameters to the radio channel conditions.

Works related to distributed and adaptive traffic scheduling can be found in [16], [17]. Both papers deal with distributed scheduling, which is a mean to provide service differentiation as well as overall network efficiency. Nonetheless, previous papers face this problem in the case of saturated traffic sources, i.e. assuming that all the stations are always in the contending state. We consider a particular traffic scenario, in which saturated stations share the bandwidth with low-rate non-saturated stations. As we detail in the next sections, this scenario is assimilable to a mixed data/real-time traffic one.

\section{Enhanced Distributed Channel ACCEss}

We assume that the reader is familiar with the IEEE 802.11 DCF standard and the EDCA extensions. Our brief description is mainly focused on the discussion of the mechanisms to dynamically adapt the MAC parameters. We consider an infrastructure network, in which an Access Point (AP) can centralize the MAC tuning operations, for both the downlink and the uplink traffic flows.

The EDCA proposal of the IEEE 802.11e Task Group is devised to differentiate the channel access probability among different traffic sources. Packets arriving to the MAC (MSDUs) are mapped into four different access categories (ACs), which represent four different levels of service for the contention to the shared medium. Each AC contends to the medium with the same rules of standard DCF, i.e., wait until the channel is idle for a given amount of inter frame space, and then access/retry following exponential backoff rules.

The access probability differentiation is provided by giving i) different Arbitration Inter-Frame Spaces AIFS, instead of the constant DIFS, and ii) different values for the minimum/maximum contention windows to be used for the backoff time extraction. Then, each $\mathrm{AC}$ is specified by the values $\operatorname{AIFS}[A C], \mathrm{CW}_{\min }[A C]$, and $\mathrm{CW}_{\max }[A C]$. The AIFS $[A C]$ values differ by an integer number of backoff slots. In particular, AIFS $[A C]=\mathrm{AIFSN}[A C] \cdot$ aSlotTime + aS IF STime, where $\operatorname{AIFSN}[A C]$ is an integer greater than 1 for normal stations and greater than 0 for APs. Separate queues are maintained in each station for different ACs and each one behaves as a single enhanced DCF contending entity.

EDCA also specifies new operation based on the concept of transmission opportunity (TXOP), which represents a time interval in which the station is authorized to hold the channel. In this work, we do not explicitly consider the TXOP differentiation since it does not affect the channel access operations but only the channel holding periods. Table 1 shows the default values of the channel access parameters defined in EDCA for 


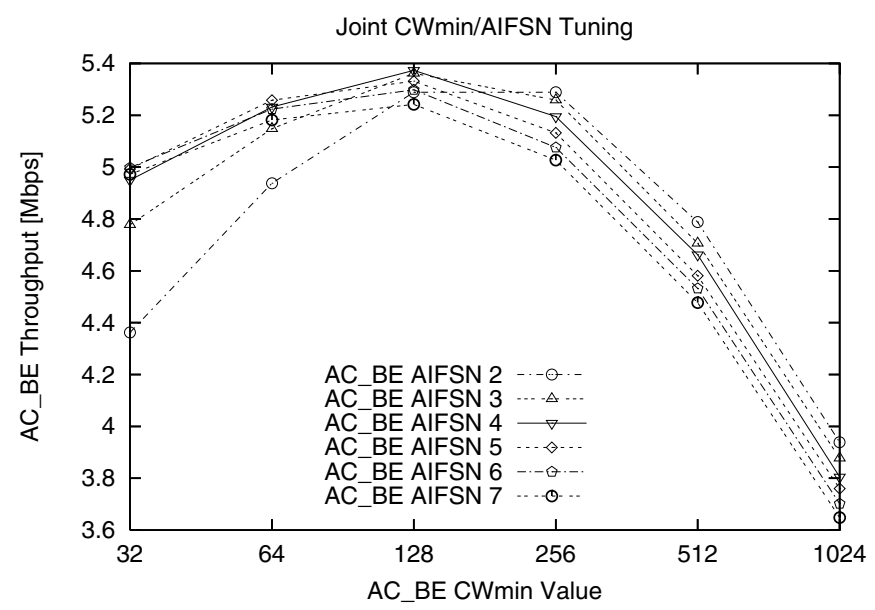

Fig. 3. Impact of $\mathrm{CW}_{\min }$ on data performance, in the case of 10 data stations, for different AIFSN values

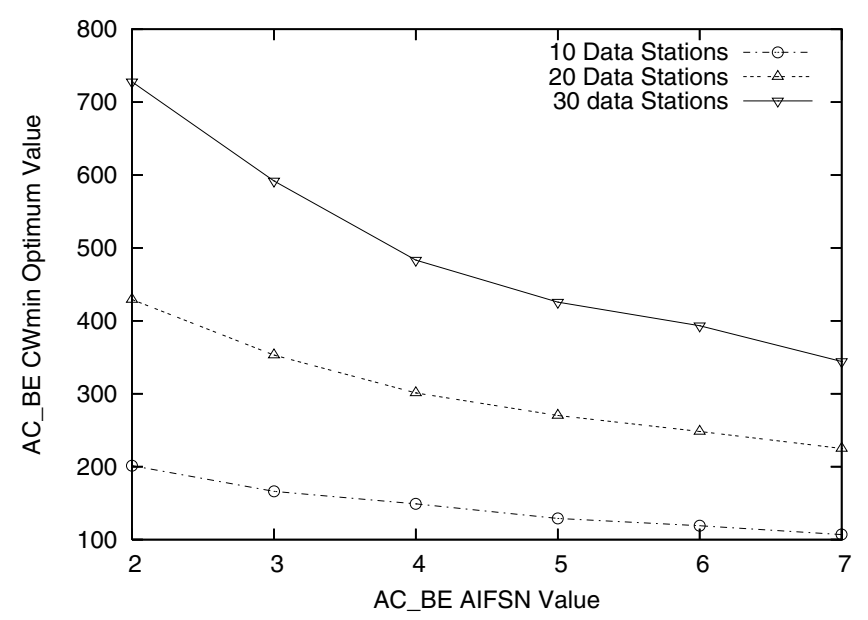

Fig. 4. Optimum $\mathrm{CW}_{\min }$ values for Data Station throughput maximization

each AC. In each beacon frame, the AP broadcasts the values of these parameters chosen for each AC. The per-class settings are specified in a special field of the beacon frame, called the EDCA Parameter Set Element. The most recent EDCA parameter set element received by the stations are used to update the appropriate MAC values.

The detailed format of the field, which is 20 bytes long, is presented in figure 2. It includes a QoS Info field, used to identify whether the EDCA parameters have changed, and four different record fields, corresponding to four different ACs. Each AC Parameter Record in turns divided into three sub-fields: the ACI/AIFSN, the ECWmin/ECWmax and TXOPLimit subfields. The first subfield contains the AIFSN bits, which indicate the number of slots to be added to the SIFS time (bit 0 to 3 ), the enabling/disabling of the admission control function (bit 4), the AC identification bits (bits 5 and 6), and a final reserved bit (bit 7). In the second subfield, bit 0 to 3 and 4 to 7 indicate, respectively, the $\mathrm{CW}_{\min }$ and $\mathrm{CW}_{\max }$ values using exponential notation with base 2 . In particular, $\mathrm{CW}_{\min }=2^{E C W \min }-1$ and $\mathrm{CW}_{\max }=2^{E C W \max }-1$, so that the minimum encoded vales is 0 and the maximum vales

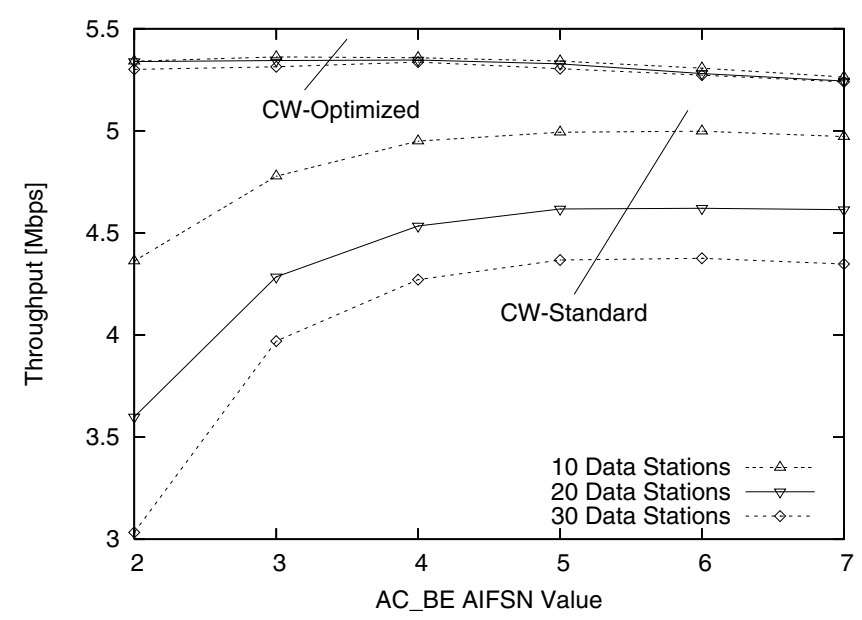

Fig. 5. Data Stations throughput vs. Data Stations AIFSN settings with and without optimized $\mathrm{CW}_{\min }$ values

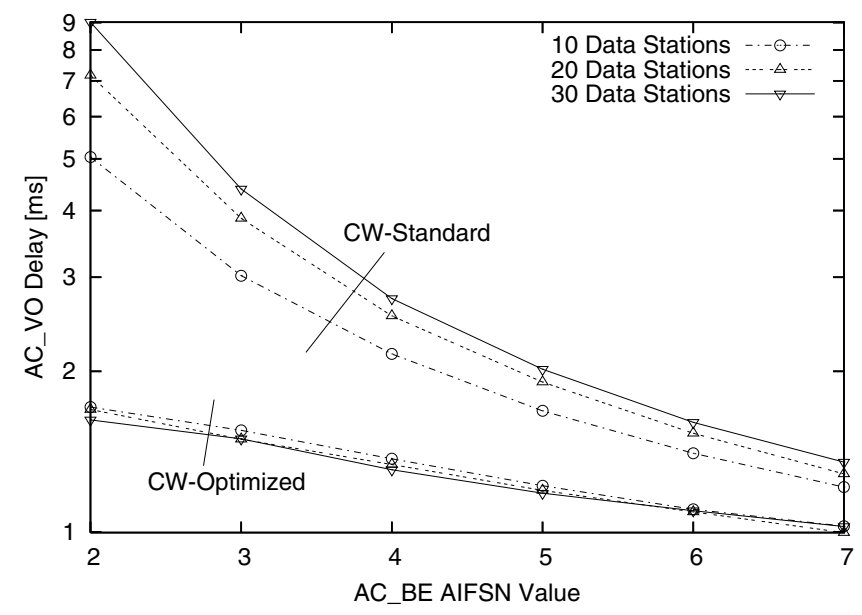

Fig. 6. Real-Time Stations delay vs. Data Stations AIFSN settings with and without optimized $\mathrm{CW}_{\min }$ values

is 32767. Finally, the last subfield, represents an unsigned integer corresponding to the TXOP value in units of $32 \mu \mathrm{s}$. The AC settings can be dynamically adapted according to the network conditions. Note that these settings only applicable to the uplink traffic, whereas the AP can use arbitrary MAC values for downlink.

\section{Performance optimization in heterogeneous TRAFFIC SCENARIOS}

\section{A. QoS problem definition}

We assume that in each station a single application is running. This corresponds to one MAC layer queue and one $\mathrm{AC}$ for each station. Two different types of MSDU sources are considered: long packet (1500 bytes) saturated sources, (i.e. the MAC layer queue is never empty) and short packet ( 80 bytes) low-rate (32 Kbps) sources (i.e. sources with packet arrival rates lower than the packet service rate). A station employing the saturated source is called data station, since this type of source corresponds to a data transfer application model. A station employing the low-rate source is called realtime station, since this type of source corresponds to a voice- 


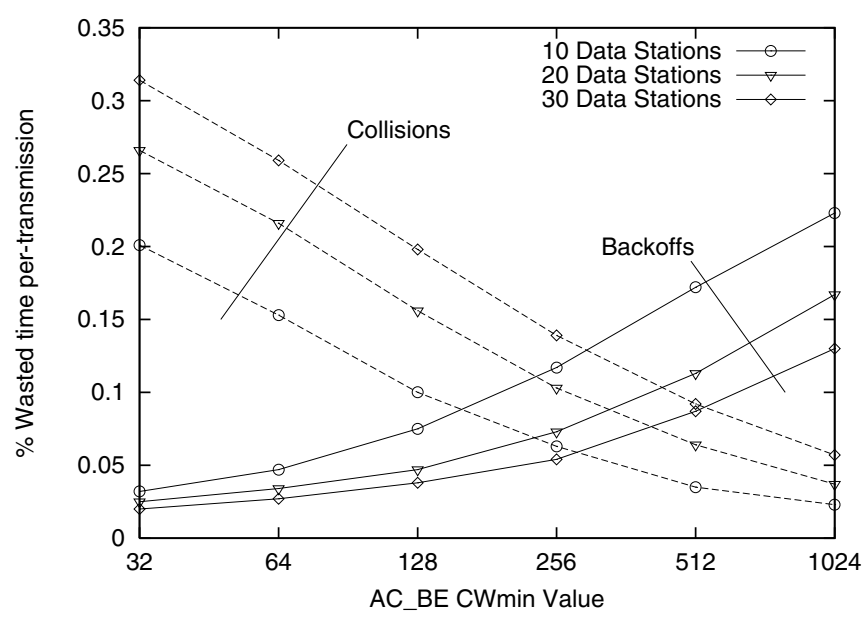

Fig. 7. Backoff expirations and collision overheads, in percentual terms, for each successful transmission vs. the AC_BE $\mathrm{CW}_{\min }$ settings, for different $\mathrm{N}$ values

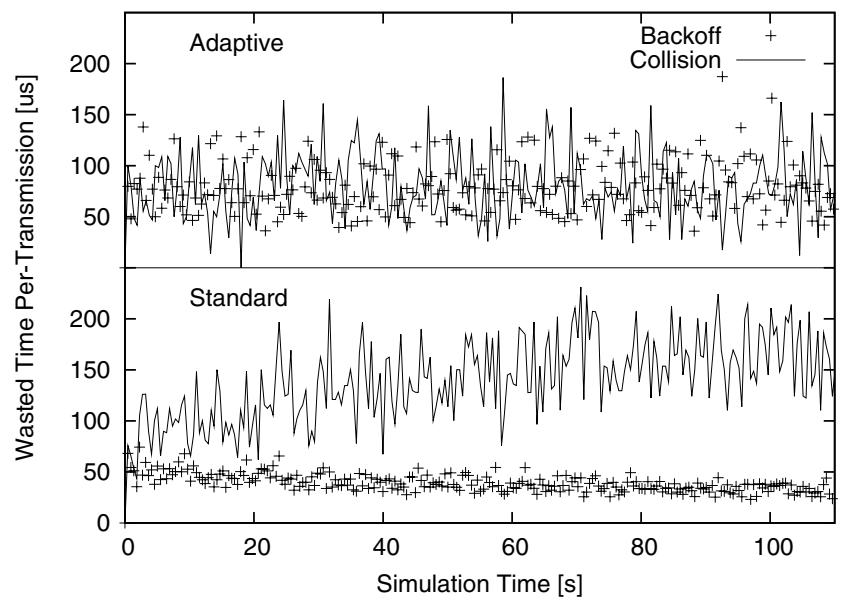

Fig. 8. Backoff expiration and collision overheads, in $\mu \mathrm{s}$, for each successful transmission vs. the AC_BE $\mathrm{CW}_{\min }$ settings, for different $\mathrm{N}$ values

like application model. Two different performance figures are used for the two different traffic sources. For the data application, the requirement is the minimization of the overall data transfer time and then the maximization of the granted bandwidth; for the real-time application, the requirement is a constraint on the average packet delivery delay.

The network scenario is represented by a fixed number of real-time (10) stations, which share the channel with a various number of $N$ data stations. We consider an $802.11 \mathrm{~b}-$ like PHY, operating at a data-rate of $11 \mathrm{Mbps}$ and a basicrate of 2 Mbps. Also, we assume that the real-time traffic is mapped onto the default AC_VO access parameters (see Fig. 1 ), whereas data sources are mapped onto the AC_BE access category, for which the MAC settings are opportunely tuned in each different QoS solution, in order to allow fast contention resolutions for real-time stations. We only focus on CW-based and AIFS-based tuning mechanisms because of their greater effectiveness in providing service differentiation in comparison to other differentiation mechanisms such as the $\mathrm{CW}_{\max }$ or the Persistence Factor (which has not been considered since

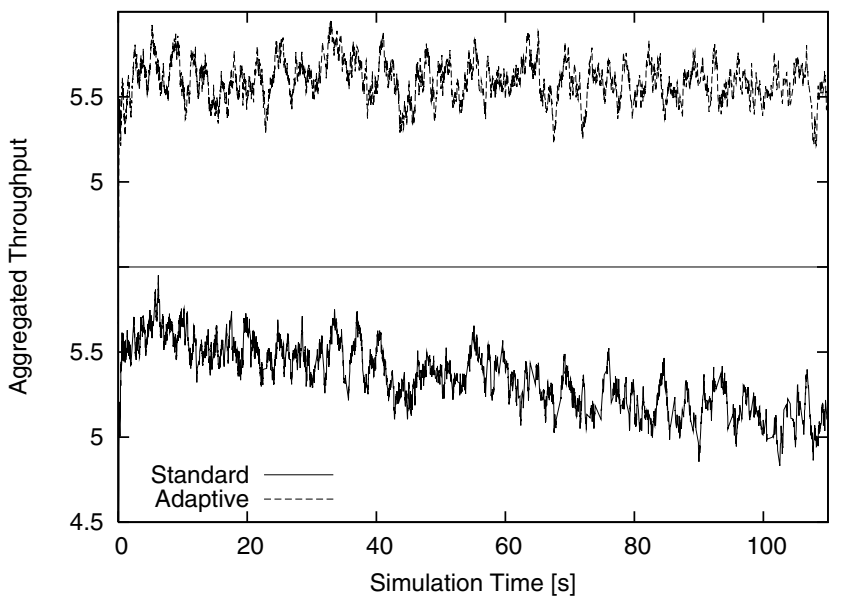

Fig. 9. Overall throughput in the case of standard and adaptive protocol as the number of data contenting stations increases

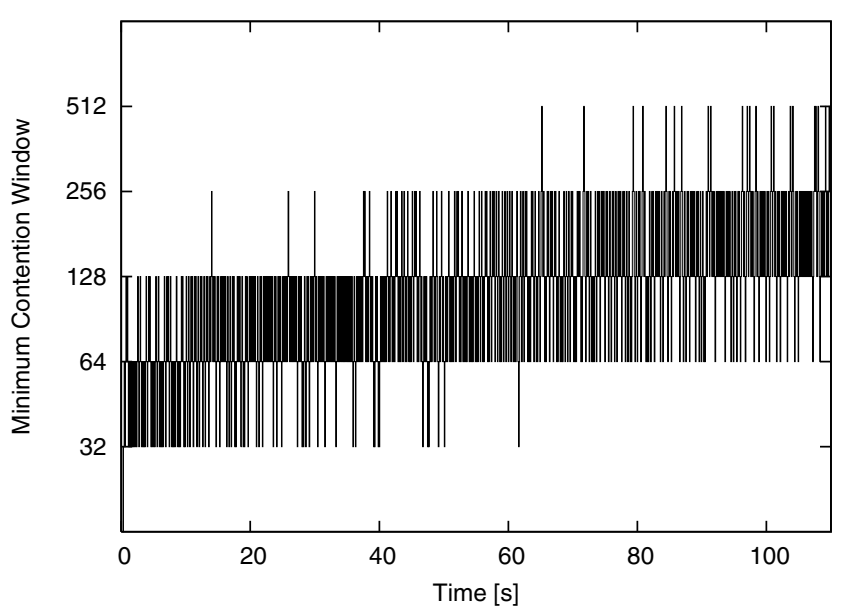

Fig. 10. Real-Time Stations delay vs. Data Stations AIFSN settings with and without optimized $\mathrm{CW}_{\min }$ values

version 5.0 of the $802.11 \mathrm{e}$ draft).

\section{B. Joint AIFSN/CW $W_{\text {min }}$ tuning}

In a previous work [18], we concluded that, in order to maximize the overall network performance, the AC_BE MAC settings should be based on an opportune configuration of both the AIFSN and $\mathrm{CW}_{\min }$ parameters. Starting from that, assuming that the AIFSN setting is able to satisfy the realtime delay requirement for a certain AC_BE $\mathrm{CW}_{\min }$ value, it is immediate to understand that higher values of this $\mathrm{CW}_{\min }$ improve the real-time performance in terms of QoS (because data stations access probability is further reduced).

Consider now Fig. 3. It shows the data stations throughput performance in the case of 10 data stations and 10 real-time stations, as the $\mathrm{CW}_{\min }$ value changes for various AIFSN values. From the figure, it is evident that the $\mathrm{CW}_{\text {min }}$ can be viewed as a possible optimization parameter for the data throughput, assumed that the real-time requirements are satisfied by the AIFSN tuning.

In the figure, it is also evident that the optimum $\mathrm{AC} \_\mathrm{BE}$ 
$\mathrm{CW}_{\min }$ value is a function of the AC_BE AIFSN value. Obviously the number of involved stations has a certain impact on the curves behavior as well. In particular, it is intuitive to understand that the larger the number of stations, the larger the $\mathrm{CW}_{\min }$ value needed to reduce the collision probability (definitively, the maxima will be all shifted towards the right side as the number of best-effort stations increases). This phenomenon is substantial to the fact that, as the AIFSN increases, the collisions among data and real-time packets get lower and lower, because most of the real-time transmissions are originated before the AC_BE AIFS expiration. Since the optimum throughput is given by the tradeoff among the minimization of the data collision probability and the resource wasted due to collisions and idle slots, it is clear that, as the real-time stations reduce their impact on the collision probability, such tradeoff is reached for lower $\mathrm{CW}_{\min }$ values.

Fig. 4 shows the optimum $\mathrm{AC} \_\mathrm{BE} \mathrm{CW}_{\min }$ values as a function of the AC_BE AIFSN settings, for different number of data stations. The optimum values have been obtained with shorter $\mathrm{CW}_{\min }$ increments, thus higher in accuracy. From the figure, we observe that as the AIFSN increases, the optimum $\mathrm{CW}_{\min }$ value tends to a limit value, which corresponds to the theoretical optimum [2], [3], achieved when only $\mathrm{N}$ data stations contend for the channel.

Fig. 5 and 6 show, respectively, the data throughput and the real-time delays, for the scenarios $\mathrm{N}=10, \mathrm{~N}=20$ and $\mathrm{N}=30$, as a function of the AC_BE AIFSN value, in the two cases of $\mathrm{CW}_{\text {min }}=32$ (standard case) and optimum $\mathrm{CW}_{\min }$ (optimal case) settings. We can see from these figures that jointly tuning the AIFSN and $\mathrm{CW}_{\min }$ parameters for the $\mathrm{AC} \_\mathrm{BE}$ traffic class allows to optimize the performance for both data (in terms of throughput) and real-time stations (in terms of delay). However, in order to productively use the optimal $\mathrm{CW}_{\text {min }}$ parameter value, for each considered traffic scenario, it is needful to know exactly the network load status.

\section{Automatic MAC PARAmeters Update ALGORITHM}

In this section we refer to the same network scenario, considering that information about the load (number of competing stations) and about the traffic sources (packet sizes and rates) is not available. After having observed that the configuration of the AC_BE minimum contention window can severely influence the total throughput, for a given AIFSN value (refer to section IV-B), we propose a simple algorithm, running at the AP, that can dynamically adapt the AC_BE AIFSN and $\mathrm{AC} \_\mathrm{BE} \mathrm{CW}_{\min }$ parameters, in order to provide the required QoS and maximize the network performance.

The tuning algorithm works as follows. If the AC_VO requirements are not satisfied: i) increase the AC_BE AIFSN value by one unit, and ii) set $\mathrm{CW}_{\min }$ to the optimal corresponding value. These operations, in turns, rely on the availability of an expression that relates the optimal $\mathrm{CW}_{\min }$ value to the AIFSN parameter and to the number of competing stations (i.e., the curves shown in figure 4).

Although some relations for the optimal $\mathrm{CW}_{\min }$ value have been derived in literature in the case of DCF and saturated stations [3], the extension to the EDCA case is not immediate. On one side, it is not possible to correctly estimate the number of per-class competing stations by simple monitoring the channel activity, such as in [19]. On the other side, in our context it is not reasonable to assume that all the stations work in saturation conditions. Thus, we propose a different approach for the optimal tuning: instead of analyzing the network status to compute the optimal $\mathrm{CW}_{\min }$ value as a function of this status, we analyze the network status to correct the MAC protocol behavior and identify the attainment of the optimal working conditions.

\section{A. Identification of the optimal working conditions}

In EDCA the channel access is managed in terms of consecutive contentions, whose winner depends on the station with the lowest backoff expiration time. However, although this channel management avoids the overheads of any polling scheme, it introduces some inefficiencies related to the contention resolution: the idle time wasted for the backoff expiration and the transmission time wasted for the collisions. The minimum contention window settings have different effects on the two sources of channel waste. On one side, as the contention window increases the collision probability is reduced because of the increment of the backoff extraction ranges. On the other side, the idle time spent for the backoff expiration grows. Thus, it exists an optimum contention window value which maximizes the throughput as a tradeoff between the increment of the backoff times and reduction of the collision times.

In [20], it is observed that this optimal condition is approximately reached whenever the backoff times are equal to the collision times (measured in terms of seconds). Refering to the considered network scenario, Fig. 7 shows the channel times wasted for backoffs and collisions versus the AC_BE $\mathrm{CW}_{\min }$. Each pair of curves refers to a different number of data stations. We do not consider $\mathrm{AC}-\mathrm{VO} \mathrm{CW}_{\min }$ as an optimization parameter since real-time stations are rarely involved in the contentions and, in any case, require very short channel access times. From the figure, we can observe that, for each pair of curves, the crossing point between the backoff times and the collision times corresponds approximately to the $\mathrm{CW}_{\min }$ value which maximize the overall throughput, as shown in [18], further confirming [20] considerations.

Also note that the collision times and the backoff times curves respectively present a decreasing and an increasing monotonic behavior in respect to the AC_BE contention window value. Thus, it is always possible to recognize whenever the current $\mathrm{CW}_{\min }$ value is higher or lower than the optimal one, by simply comparing the two sources of wasted times. Specifically, if the collision time is higher than the backoff time, it is necessary to further reduce the collision probability by increasing the $\mathrm{CW}_{\min }$ parameter. Conversely, if the backoff time is higher than the collision time, it is necessary to avoid unused idle times by decreasing the $\mathrm{CW}_{\text {min }}$ values.

In principle, this comparison can be performed by the AP on a per-beacon basis and allows to incrementally correct 
the AC_BE contention window values, without requiring any network load estimator and any preliminarily assumption about the data sources. The capability to optimize the $\mathrm{CW}_{\min }$ value by tracking the network dynamics, in the case of station activations/deactivations or in the case of not-saturated data stations, clearly depends on the beacon interval value.

\section{B. Dynamic contention window corrections algorithm}

Starting from the above considerations, we propose a very simple correction algorithm for the AC_BE minimum contention window. During each beacon interval $i$, the AP counts the overall time $B_{i}$ spent in backoffs and the overall time $C_{i}$ spent in collisions. Then, it updates the AC_BE $\mathrm{CW}_{\min }$ value as follows:

$$
\begin{gathered}
C W_{\text {min }}(i)=C W_{\text {min }}(i-1) \cdot 2 \quad C_{i}>B_{i} \\
C W_{\text {min }}(i)=\max \left(32, C W_{\text {min }}(i-1) / 2\right) \quad C_{i} \leq B_{i}
\end{gathered}
$$

The new contention window values is finally broadcasted through the EDCA parameter set field. No filtering operation on the channel wasted times and no hysteresis for the contention window updates are considered.

Fig. 8 shows an example of the algorithm effects in a dynamic load scenario. The figure has been obtained with our simulator, by initializing the network with 10 real-time stations and 5 data stations. After the simulation begins, new data stations join the network at intervals of 10 seconds. The simulation ends when the 11-th data station arrives. The beacon time is set to $0.1 \mathrm{~s}$. In the figure we compare the channel wasted times in the case of standard protocol (lower trace), with $\mathrm{CW}_{\min }$ set to 32 , and in the case of adaptive contention window tuning (upper trace). In order to improve the figure readability, the collision times and the backoff times are plotted at intervals of 5 beacons. From the figure we see that, in the case of fixed contention window setting, as the number of station increases (i.e., as the simulation time advances) the collisions wastes more and more resources, while the backoff times are slightly reduced. Indeed, our adaptive algorithm allows to equalize these wasted times.

In Fig. 9 we notice that this operation really corresponds to the maximization of the overall network throughput. In fact, in the case of the adaptive protocol the overall throughput does not suffer the increment of the number of competing stations and remains, averagely, around the maximum values. Finally, in Fig. 10 we show the temporal updates of the AC_BE minimum contention windows. Since we do not used any $C_{i}-$ $B_{i}$ threshold for triggering the contention updates, we can see that the $\mathrm{CW}_{\min }$ values can change beacon by beacon. However, the network performance does not degrade because of the fast changes, while the protocol results are very quickly adapted to the station dynamics.

\section{CONCLUSIONS}

In this paper we analyze the influence of the joint use of AIFSN and $\mathrm{CW}_{\text {min }}$ parameters settings in the case of heterogeneous traffic sources, focusing on the possibility to optimize network resources. We discuss and evaluate a very simple solution for adapting these MAC parameters to the network congestion status, using incremental corrections driven by the monitoring of the channel occupancy status. From simulation results, we have concluded that a joint utilization of the AIFSN and $\mathrm{CW}_{\min }$ parameters results in an optimal and effective solution for optimizing the overall system performance, thus achieving the delay constraints of real-time stations, while maximizing the throughput for data stations.

\section{REFERENCES}

[1] EEE 802.11 WG, IEEE Std 802.11, 1999 edition. International standard for Information Technology. Telecommunications and information exchange between systems - Local and metropolitan area networks. Specific Requirements Part 11: Wireless LAN Medium Access Control (MAC) and Physical Layer (PHY) specifications, 1999.

[2] G. Bianchi, "Performance analysis of the IEEE 802.11 distributed coordination function," IEEE JSAC, Vol.18, No.3, Mar 2000, pp. 535-47.

[3] F.Cali', M.Conti and E.Gregori, " Dynamic IEEE 802.11: Design, Modeling and and Performance Evaluation,"IEEE JSAC, Vol.18, No.9, Sept. 2000, pp. 1774-1786.

[4] M. Heusse, F. Rousseau, G. Berger-Sabbatel, and A. Duda, "Performance anomaly of $802.11 \mathrm{~b}$ ", in Proc. of IEEE INFOCOM 03, San Francisco, CA, April 2003.

[5] EEE 802.11 WG, IEEE Std 802.11e/D8.0, Draft Supplement to IEEE standard for Telecommunications and Information exchange between systems. Local and metropolitan area networks. Specific Requirements Part 11: Wireless LAN Medium Access Control (MAC) and Physical Layer (PHY) specifications, February 2004.

[6] Lamia Rhomdani, Qiang N, Thierry Turletti, "AEDCF: Enhanced Service Differentiation for IEEE 802.11 Wireless Ad-Hoc Networks", INRIA technical report RR4544,September 2002.

[7] I. Aad, C. Castelluccia, "Remarks on Per-Flow Differentiation in IEEE 802.11", In Proceedings of European Wireless 2002.

[8] Sunghyun Choi, Javier del Prado, Stefan Mangold, and Sai Shankar, "IEEE 802.11e Contention-Based Channel Access (EDCF) Performance Evaluation", Proc. IEEE ICC 03, Anchorage, Alaska, USA, May 2003.

[9] S. Mangold, S. Choi, P. May, O. Klein, G. Hiertz, L. Stibor, "IEEE 802.11e Wireless LAN for Quality of Service", In Proc. of the European Wireless, Florence, Italy, February 2002.

[10] G. Bianchi, I. Tinnirello, "Analysis of Priority Mechanisms based on Differentiated Inter-Frame Spaces in CSMA/CA", in Proc. IEEE VTC 2003, Orlando (FL), October 2003.

[11] J.W. Robinson, T.S. Randawa, "Saturation throughput analysis of IEEE 802.11e enhanced sitributed coordination function", IEEE JSAC 2004, vol. 22, n.5, pp. 912-928.

[12] Sven Wiertholter, Christian Hoene, "Design and Verification of an IEEE 802.11e EDCF simulation model in ns-2.26", November 2003.

[13] Qiang Ni, Lamia Rhomdani, Thierry Turletti and Imad Aad, "QoS Issues and Enhancements for IEEE 802.11 WLAN", INRIA technical report RR4612, November 2002

[14] L. Bononi, M. Conti, E. Gregori, "Runtime Optimization of IEEE 802.11 Wireless LANs Performance," IEEE Trans. on Parallel and Distributed Systems, Vol.15, No.1, Jan. 2004, pp. 66-80.

[15] M. Barry, A. T. Campbell, A. Veres, "Distributed Control Algorithms for Service Differentiation in wireless Packet Networks", Proc. of IEEE INFOCOM 2001, pp. 582-590.

[16] V. Kanodia, C. Li, A. Sabharwal, B. Sadeghi, and E. Knightly, "Distributed Priority Scheduling and Medium Access in Ad Hoc Networks", ACM WINET journal, September 2002.

[17] N. H. Vaidya, P. Bahl, and S. Gupta, "Distributed Fair Scheduling in a Wireless LAN", iProc. of MobiCom 2000, Boston, Massachusetts, USA, August 2000.

[18] L.Scalia, I.Tinnirello, "Differentiation Mechanisms for Heterogeneous Traffic Integration in IEEE 802.11 Networks", Proc. of BroadWim04, October 2004, San Jose', CA. US.

[19] G.Bianchi, I. Tinnirello, "Kalman Filter Estimation of the Number of Competing Terminals in an IEEE Network", in Proc. of Infocom 2003, pp. 844-852, vol. 2, San Francisco, CA, March 2003.

[20] R.G. Gallagher, "A perspective on Multiaccess Channels", IEEE Trans. Information Theory, vol. 31, no. 2, pp. 124-142, Mar. 1985. 